\title{
Long-range adsorbate interactions mediated by a two-dimensional electron gas
}

\author{
N. Knorr, ${ }^{1,2}$ H. Brune, ${ }^{1}$ M. Epple, ${ }^{1}$ A. Hirstein, ${ }^{1}$ M. A. Schneider, ${ }^{1,2}$ and K. Kern ${ }^{1,2}$ \\ ${ }^{1}$ Institut de Physique Expérimentale, Ecole Polytechnique Fédérale de Lausanne, CH-1015 Lausanne, Switzerland \\ ${ }^{2}$ Max-Planck-Institut für Festkörperforschung, Heisenbergstrasse 1, D-70569 Stuttgart, Germany
}

(Received 2 November 2001; published 8 March 2002)

\begin{abstract}
We report on long-range interactions between adsorbates on metal surfaces with a surface state. A comparison of three adsorbate/substrate systems $[\mathrm{Cu} / \mathrm{Cu}(111), \mathrm{Co} / \mathrm{Cu}(111)$, and $\mathrm{Co} / \mathrm{Ag}(111)]$ suggests the general existence of such interactions and shows up common characteristics. In all cases, the interaction energy $E(r)$ manifests itself up to a distance of $60 \AA$, decays as $1 / r^{2}$, and oscillates with a period of $\lambda_{\mathrm{F}} / 2$. Our data are in excellent agreement with theory and establish the link between the spatial variation of the interaction energy and the adsorbate scattering properties. We demonstrate that the long-range interactions stabilize an ordered two-dimensional (2D) gas of adsorbates and thus create states of dilute 2D matter.
\end{abstract}

DOI: 10.1103/PhysRevB.65.115420

PACS number(s): 68.35.Fx, 68.37.Ef, 68.43.Jk, 81.16.Dn

Lateral adsorbate-adsorbate interactions have attracted theoretical $^{1}$ and experimental ${ }^{2}$ interest since the 1970 s. The interactions have several origins that can be divided according to the mutual adsorbate separation. At small interatomic distances direct electronic interaction dominates leading to the formation of localized chemical bonds. This interaction falls off exponentially and is, therefore, of very short range. At larger separations adsorbate interactions are predominantly indirect and may be mediated in three ways: electrostatically (dipole-dipole) and elastically (deformation of substrate lattice), which both lead to nonoscillatory interactions that decay monotonically with separation $r$ as $1 / r^{3}{ }^{3}$ The third way of mediation, which is the subject of the present study, is by substrate electrons leading to an oscillatory interaction energy.

A foreign atom dissolved in a solid, or adsorbed on a surface, imposes its potential onto the host electrons, which they screen by density oscillations. Friedel introduced such oscillations with wave vector $2 k_{\mathrm{F}}$ to calculate the conductivity of dilute metallic alloys. ${ }^{4}$ Whereas Friedel oscillations in the bulk escape from direct observation, they become apparent at the surface. ${ }^{5}$ Scanning tunneling microscopy (STM) images taken at low bias directly reflect the oscillating quantity, namely, the local density of states close to $E_{\mathrm{F}}$, enabling a direct observation of Friedel oscillations. The first case was reported for carbon atoms adsorbed on $\mathrm{Al}(111){ }^{6}$ The original data are reproduced in Figs. 1(a) and 1(b) for contrasting Friedel oscillations of bulk electrons with those of surfacestate electrons [Figs. 1(c) and 1(d)]. In Fig. 1(a) two C atoms appear as localized protrusions. In Fig. 1(b) the same area is scanned at smaller tip-sample distance, which has the effect that the $\mathrm{C}$ atoms become transparent. This reveals the redistribution of substrate electrons particularly clearly. Carbon withdraws charge from the three nearest $\mathrm{Al}$ neighbors, which, therefore, appear darker $(\Delta z=-0.34 \AA)$. Due to Friedel oscillations, the next-nearest $\mathrm{Al}$ neighbors accumulate charge and appear brighter $(\Delta z=+0.18 \AA)$.

Inspecting Figs. 1(a) and 1(b) one realizes that adsorbates may interact via Friedel oscillations through the fact that the binding energy of one adsorbate depends on the substrate electron density, which oscillates around the other adsorbate. Lau and Kohn predicted such oscillatory interactions to de- pend on distance as $\cos \left(2 k_{\mathrm{F}} r\right) / r^{5} \cdot{ }^{7}$ Hence the bulk, threedimensional (3D), electron mediated adsorbate interactions fall off much faster than the dipole-dipole and elastic interactions. The superposition of all three indirect interactions, dipole-dipole, elastic, and bulk-electron mediated, leads to complicated behavior with high chemical specificity. For their investigation, STM has emerged as a quantitative tool, ${ }^{8}$ supplementing the well-established field-ion-microscopy method. ${ }^{2,9}$

While the range of all interactions discussed above never goes beyond a few atomic distances, Lau and Kohn predicted that screening in a $2 \mathrm{D}$ electron system leads to a much slower fall off, $\cos \left(2 k_{\mathrm{F}} r\right) / r^{2}$, and thus to interactions with extremely long range. ${ }^{7}$ The required $2 \mathrm{D}$ nearly free-electron gas is realized in Shockley-type surface states of closepacked surfaces of noble metals. These states are located in narrow band gaps in the center of the first Brillouin zone of the (111)-projected bulk band structure. Thus they have extremely small Fermi wave vectors and consequently the Friedel oscillations of the surface state have a significantly larger wavelength than those of bulk states. Figure 1(c) shows an example for $\operatorname{Ag}(111)\left[k_{\mathrm{F} \text {.surf }}=0.083 \AA^{-1}\right.$ (Ref. 10) and $k_{\mathrm{F} \text {,bulk }}=1.2 \AA^{-1}$ (Ref. 11)] with two substitutional defects appearing as protrusions on the otherwise clean surface. There is a smooth modulation in the apparent height of the $\mathrm{Ag}$ atoms extending over the entire image. These are the surface-state Friedel oscillations, ${ }^{12}$ which are readily detectable up to a distance of more than $100 \AA$ in the large-scale STM image shown in Fig. 1(d).

The first experimental indication of long-range interactions, possibly mediated by surface-state Friedel oscillations, came from equidistant bulk segregated impurities on $\mathrm{Cu}(111) .{ }^{13}$ The first quantitative interaction energies were recently reported by Repp et al. for the system $\mathrm{Cu} / \mathrm{Cu}(111) .{ }^{14}$

The results of Ref. 14 clearly show the predicted oscillation period of $\lambda_{\mathrm{F}} / 2$ and the $1 / r^{2}$ decay for large distances. However, there are significant deviations from theory in the distance regime where the interactions are expected to be strongest, namely, at $r<20 \AA$. Quantitative experimental information down to short distances is crucial for two reasons. First, the interactions at these distances become sufficiently strong to delay nucleation and to stabilize ordered 2D gases of adsorbates. Second, experimental data on this length scale 
(a)

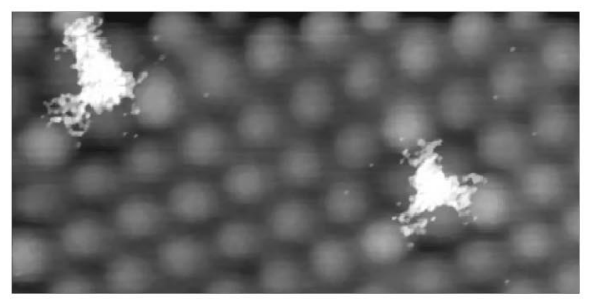

(b)

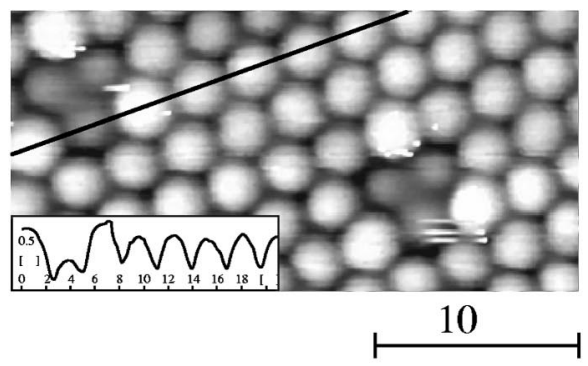

(c)
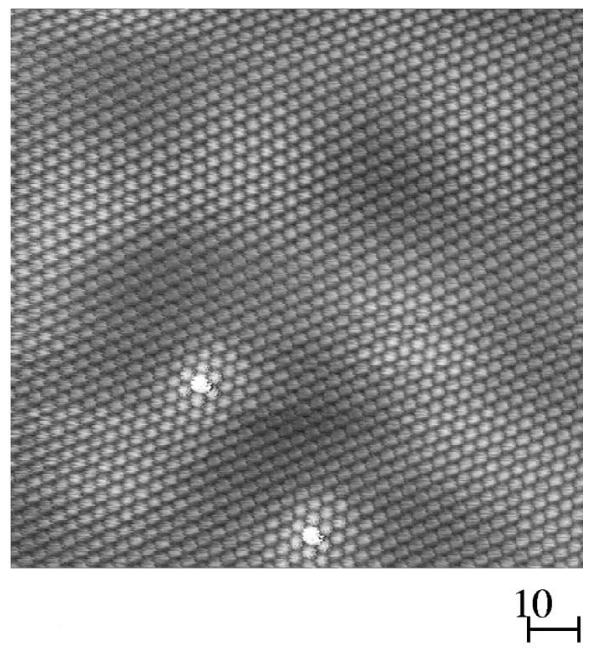

(d)

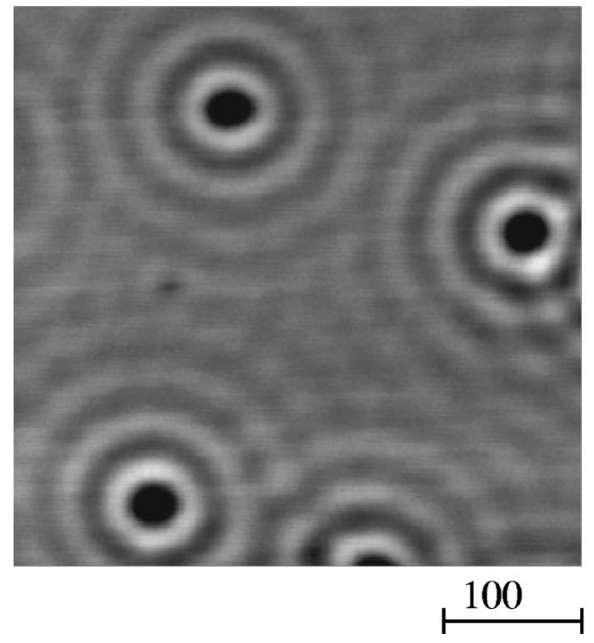

FIG. 1. (a), (b) Two $\mathrm{C}$ atoms chemisorbed on $\mathrm{Al}(111)$ imaged at different tip-sample distances $\left[\right.$ (a) $V_{\mathrm{t}}=-70 \mathrm{mV}$, (b) $V_{\mathrm{t}}$ $=-20 \mathrm{mV}$, (a), (b) $\left.I_{\mathrm{t}}=41 \mathrm{nA}, T=300 \mathrm{~K}\right]$. (c) Two substitutional defects on $\operatorname{Ag}(111)\left(V_{\mathrm{t}}=-5 \mathrm{mV}, I_{\mathrm{t}}=8 \mathrm{nA}, T=9 \mathrm{~K}\right)$. (d) Large scale image of the same surface as in (c) showing the long-range oscillations with $\lambda=\pi / k_{\mathrm{F}}=38 \AA$ around four point defects on $\operatorname{Ag}(111)\left(V_{t}=24 \mathrm{mV}, I_{t}=0.5 \mathrm{nA}, T=9 \mathrm{~K}\right)$. (a)

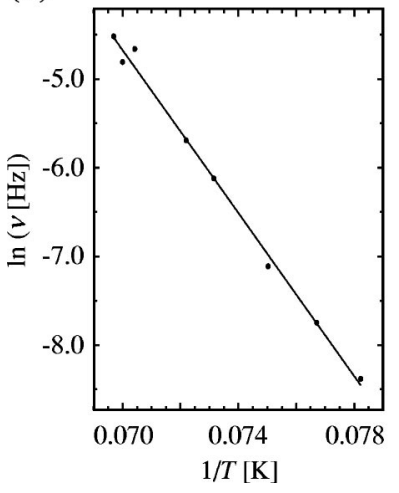

(b)

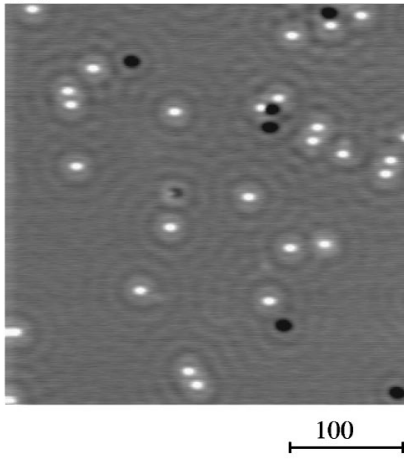

FIG. 2. (a) Arrhenius plot of the jump rate of isolated $\mathrm{Cu}$ monomers. The STM tip had no influence on diffusion parameters for gap resistances $R_{\mathrm{t}}>1 \times 10^{8} \Omega$. (b) Excerpt from time sequence of STM images recorded to trace diffusing $\mathrm{Cu}$ atoms on $\mathrm{Cu}(111)(2 \mathrm{~min} /$ frame, $T=13.5 \mathrm{~K}, \Theta=1.4 \times 10^{-3} \mathrm{ML}, V_{\mathrm{t}}=100 \mathrm{mV}, I_{\mathrm{t}}=0.1 \mathrm{nA}$; see Ref. 18).

are important for comparison with theory since recent density-functional-theory calculations ${ }^{15,16}$ can now address this distance range, whereby they meet the validity range of scattering theory. ${ }^{17}$

In the present study we remove the disagreement with theory for $\mathrm{Cu} / \mathrm{Cu}(111)$ by careful analysis of our data in terms of two-body interactions only. Furthermore, by investigating the interactions also for Co atoms on $\operatorname{Ag}(111)$ we observe the oscillation wavelength to change according to the surface-state band structure of the respective substrate. This unambiguously identifies the cause of the interaction as mediation by surface-state electrons. Comparing different adsorbates, $\mathrm{Cu}$ and $\mathrm{Co}$ atoms, on the same substrate, $\mathrm{Cu}(111)$, reveals the insensitivity to the adsorbate's chemical identity and suggests the general existence of long-range interactions on surface-state substrates.

We deposited $\mathrm{Cu}$ and $\mathrm{Co}$ from thoroughly degassed filaments (99.996\%) onto well-prepared $\mathrm{Cu}(111)$ and $\mathrm{Ag}(111)$ surfaces held inside a low-temperature STM contained in ultrahigh vacuum and cooled to $6 \mathrm{~K} .{ }^{10}$ After deposition of $1.4 \times 10^{-3} \mathrm{ML}$ of $\mathrm{Cu}$ onto $\mathrm{Cu}(111)$, the surface showed randomly distributed, immobile $\mathrm{Cu}$ adatoms. To find the temperature range where the interaction is observable and to measure the corrugation of the adsorbate's potential-energy surface, we first studied the diffusion rate $\nu$ of isolated $\mathrm{Cu}$ monomers as a function of time and temperature by tracing their trajectory on consecutive STM images taken from the same surface area [see Fig. 2(b)].

To simplify the analysis, we took care that the temperature was low enough for the average jump rate to stay below the image recording rate. The jump rate shows perfect Arrhenius behavior [Fig. 2(a)] from which we derive the energy barrier and attempt frequency for $\mathrm{Cu} / \mathrm{Cu}(111)$ tracer diffusion to be $E_{m}=(40 \pm 1) \mathrm{meV}$ and $\nu_{0}=1$ $\times 10^{12.0 \pm 0.5} \mathrm{~s}^{-1}$, respectively. The experimental energy barrier is within the error of the previously reported value, ${ }^{14}$ and compares well with recent $a b$ initio calculations. ${ }^{15}$ We observe only jumps by entire lattice spacings, indicative of a significant binding-energy difference between the two three- 

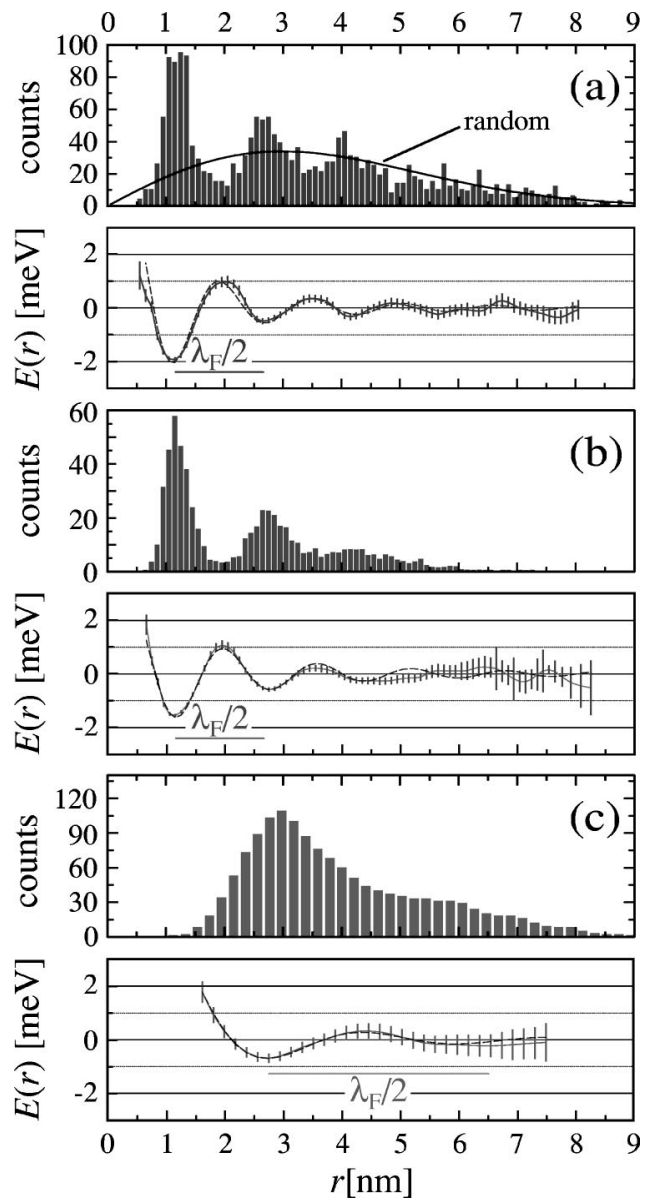

FIG. 3. Histogram of (a) $\mathrm{Cu} / \mathrm{Cu}(111)$, (b) $\mathrm{Co} / \mathrm{Cu}(111)$, and (c) $\mathrm{Co} / \mathrm{Ag}(111) \mathrm{NN}$ distances $\left[(\mathrm{a}) \Theta=1.4 \times 10^{-3} \mathrm{ML}, T=15.6 \mathrm{~K}\right.$; (b) $\Theta=2 \times 10^{-3} \mathrm{ML}, T=10.2 \mathrm{~K}$; (c) $\Theta=4 \times 10^{-4} \mathrm{ML}, T=18.5 \mathrm{~K}$ ]. Fits of our data with the nonperturbative result of Ref. 17 are shown as dashed lines. The fits have been performed with $c=10 \AA$ in (a), $14 \AA$ in (b), and $3 \AA$ in (c).

fold hollow sites. In agreement, theory finds the hcp site to be as unstable as the bridge site and therefore predicts diffusion to occur between fcc sites only. ${ }^{15}$

We quantify the lateral adatom interaction by analyzing extensive STM time sequences in terms of site-occupation probabilities as a function of adatom distances. In order to focus on two-body interactions we counted only nearestneighbor (NN) distances $r$, i.e., a distance $r$ from a selected atom to a nearby atom is counted only if no third scatterer (adatom or impurity) is closer-than $r$. For the low adatom coverages $\left(\Theta=1 \times 10^{-3} \mathrm{ML}\right)$ and the low defect densities $\left(\Theta=2 \times 10^{-4} \mathrm{ML}\right)$ this is a good approximation to the idealized situation of two isolated, interacting adatoms. A distance histogram $f(r)$ obtained that way from an STM series recorded at $15.6 \mathrm{~K}$ [Fig. 3(a)] shows significant oscillatory deviations from random site occupation $f_{\text {ran }}(r)$, shown as a solid curve. One finds the following expression for $f_{\text {ran }}(r)$ at coverage $\Theta$, image size $L \times L$, with $N$ $=L^{2} \Theta / A_{\text {unit cell }}$ atoms per image and a set of $n$ images:

$$
\begin{aligned}
f_{\operatorname{ran}}(r)= & \left(2 \pi r \Delta r n N^{2} / L^{2}\right)\left(1-\pi r^{2} / L^{2}\right)^{N} \\
& \times\left(\left[\pi L^{2}+(4-\pi) r^{2}-4 r L\right] / \pi L^{2}\right) .
\end{aligned}
$$

The first term is the linearly increasing probability of finding a pair of atoms a distance $r$ apart (the width of the histogram classes is $\Delta r$ ), the second is the probability of finding such a pair with no third atom in the area $\pi r^{2}$ around the first, and the third term accounts for the finite image size. This function was tested successfully with kinetic Monte Carlo (KMC) simulations for statistical growth. Boltzmann statistics yields the differences in adatom binding energy $E(r)$ $=-k_{\mathrm{B}} T \ln \left[f(r) / f_{\text {ran }}(r)\right]$. The curve shown below the histogram is the result of an average of $E(r)$ curves obtained from histograms at various temperatures $(14.3<T$ $<16.2 \mathrm{~K})$. The reason for including a range of temperatures is that at higher $T$, the statistical independence of consecutive images is sufficient also for the adsorbates bound in the first profound minimum, whereas the smaller energy variations in the tail of the interaction can be studied better at lower $T$.

The $E(r)$ curve for $\mathrm{Cu} / \mathrm{Cu}(111)$ clearly shows oscillations in binding energy extending up to $60 \AA$. For $r>20 \AA, E(r)$ behaves as in Ref. 14. However, our data agree with the scattering theory ${ }^{17}$ way down to the first minimum in the interaction energy, where the former study $^{14}$ found a decrease of the interaction strength. From the published histograms, and from the number of distances analyzed, we conclude that the authors of Ref. 14 analyzed all interatomic distances and, therefore, included many-body interactions, which explains the difference with the present study.

For a quantitative comparison with theory we use the model by Hyldgaard and Persson ${ }^{17}$ establishing a link of $E(r)$ with the scattering properties of the adsorbates. The fit reveals excellent agreement between theory and experiment. To extend the model to small values of $r$ we introduced a parameter $c$ as follows: $E(r)=-A \mathrm{E}_{0}(2 \sin \delta / \pi)^{2} \sin (2 q r$ $+2 \delta) /\left[(q r)^{2}+(q c)^{2}\right]$. We note that identical values for the adsorbate's scattering phase $\delta$ and for the wave vector $q$ are obtained when fitting without $c$ and starting from the first maximum of $E(r)$. The position of the first minimum determines the scattering phase $\delta=(0.50 \pm 0.07) \pi$ and a scattering amplitude of $A=0.13 \pm 0.01$, which are in good agreement with the properties expected of a black-dot scatterer. The best fit is obtained with $c=10 \AA$, meaning that the first minimum is slightly attenuated with respect to a $1 / r^{2}$ behavior, which may be indicative of the onset of repulsive interactions at short distances. Notice, however, that the first minimum of $E(r)$ is clearly more attractive than the second one in contrast to Ref. 14. The wave vector $q=(0.20 \pm 0.01) \AA^{-1}$ is in good agreement with the band structure of the $\mathrm{Cu}(111)$ surface state $\left[k_{\mathrm{F}}=0.21 \AA^{-1}\right.$ (Ref. 12)].

For short distances, there is a strong repulsion between the $\mathrm{Cu}$ adatoms before they become bound to each other in a dimer at one nearest-neighbor distance [the dimer bond energy was calculated to be $E_{\mathrm{b}}=520 \mathrm{meV}$ (Ref. 19)]. This short-range repulsion is characterized by the height $E_{\max }$ and location $r_{\max }$ of the maximum energy difference of threefold binding sites as a function of the distance between two adatoms. The repulsion is evidenced by the following observations permitting to establish lower bounds of $E_{\max }$ by comparison with KMC simulations. The absence of dimer formation at $16.5 \mathrm{~K}$ after $20 \mathrm{~min}$ observation time $\left(\Theta=1.4 \times 10^{-3} \mathrm{ML}\right.$, image size $\left.800 \times 800 \AA^{2}\right)$ yields 
$E_{\max } \geqslant 13 \mathrm{meV}$. The observed absence of dimer formation, beyond the few ones expected from statistical growth, for deposition of $3 \times 10^{-3} \mathrm{ML}$ at $19 \mathrm{~K}$ equally yields $E_{\max }$ $\geqslant 13 \mathrm{meV}$. From the observation of the onset of dimer formation at $19-21 \mathrm{~K}$ reported in Ref. 14, one derives 10-16 $\mathrm{meV}$ as an upper bound for $E_{\max }$ under the assumptions of $\Theta=3.0 \times 10^{-3} \mathrm{ML}$ and that "onset of dimer formation" corresponds to $10 \%$ of the monomers having formed dimers and trimers after $2 \mathrm{~min}$. In ab initio calculations a slightly larger value of $E_{\max } \approx 40 \mathrm{meV}$ has been reported..$^{15}$ For reasons of scale on the energy axis we did not include this short-range repulsion in Fig. 3, but we note its importance in delaying nucleation to much higher coverages than in classical nucleation and growth scenarios, ${ }^{15,16,19,20}$ which can explain the small apparent diffusion prefactors systematically deduced for systems with small barriers. ${ }^{20}$

To investigate how the long-range interactions relate to the adsorbate's chemical nature, we explored pair correlations in the very same way as for $\mathrm{Cu}$ also for $\mathrm{Co}$ adatoms on $\mathrm{Cu}(111)$ [Fig. 3(b)]. The results are within error bars identical to $\mathrm{Cu} / \mathrm{Cu}(111): \delta=(0.49 \pm 0.03) \pi, A=0.12 \pm 0.01$, and $q=(0.20 \pm 0.01) \AA^{-1}$, and the interaction decays again exactly as $1 / r^{2}$. This suggests the generality of surface-statemediated interactions between adsorbates.

To prove their electronic origin we have investigated the interaction period for $\mathrm{Co}$ on $\mathrm{Ag}(111)$, which has a different surface-state band structure resulting in an expected $E(r)$ period of $\lambda_{\mathrm{F}} / 2=38 \AA .^{10}$ The wavelength of the interactions is more than twice as long, as is evident from the $E(r)$ curve [Fig. 3(c), the fit yields $q=(0.10 \pm 0.02) \AA^{-1}$, in agreement with $k_{\mathrm{F}}=0.083 \AA^{-1}$ ]. Co atoms repel each other for $r$ $<20 \AA$ and the first minimum in interaction energy is at around $27 \AA$. Note that the scattering phase shift is with $\delta=(0.33 \pm 0.02) \pi$, smaller than in the above examples. Also the absolute values for $E(r)$ are much smaller. We attribute this to the lower total electron density in the surface state of $\mathrm{Ag}$ as compared to $\mathrm{Cu}$ since the surface-state band edge $\mathrm{E}_{0}$ of $\operatorname{Ag}(111)$ is closer to $E_{\mathrm{F}}$, while the density of states $L_{0}$ $=m^{*} / \pi \hbar^{2}$ is roughly the same for both surfaces due to their similar effective masses $m^{*}$.

The scaling of the interaction period with $\lambda_{\mathrm{F}}$ of the surface state clearly establishes that the observed long-range interactions are mediated by the nearly free $2 \mathrm{D}$ electron gas of the surface state and excludes mediation via elastic lattice deformation. In addition, our results enable quantitative comparison with theory; the short-range data agree reasonably well with recent $a b$ initio results; ${ }^{15}$ in the long range we confirm the theory of Lau and Kohn and the model derived from it by Hyldgaard and Persson. As opposed to short-range interactions the surface-state-mediated long-range interactions are shown to be far less element specific and, therefore, of general significance since they predominantly reflect the surface-state band structure. Despite the fact that the observed interaction energies are small, they are expected to influence every adsorbate/substrate system with small diffusion barriers.

Let us now turn to an important implication of the observed long-range interactions. They may lead to long-range
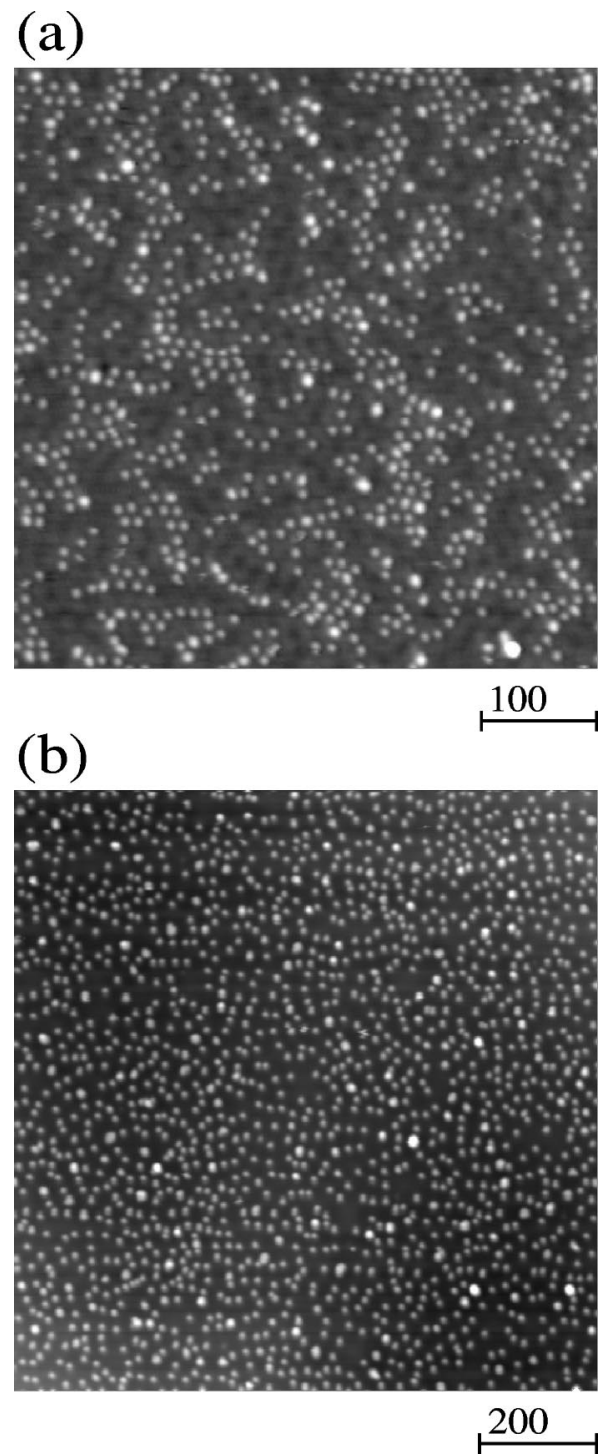

FIG. 4. Attempts to create ordered superlattices. (a) The scattering phase of $\delta=\pi / 2$ for $\mathrm{Co} / \mathrm{Cu}(111)$ favors atomic chains but inhibits hexagonal lattices $\left(\Theta=2.1 \times 10^{-2} \mathrm{ML}, T_{\mathrm{ads}}=19 \mathrm{~K}\right)$. (b) For Co/ $\operatorname{Ag}(111), \quad \delta=\pi / 3$ favoring hexagonal lattices, however, the interactions are too weak to establish long-range order $(\Theta=1.2$ $\left.\times 10^{-2} \mathrm{ML}, T_{\text {ads }}=19 \mathrm{~K}\right)$.

order between molecular and atomic adsorbates, which might be exploited in manifold ways. ${ }^{21}$ Here we consider the creation of two-dimensional solids with a lattice constant given by the first minimum of the interaction energy. While the degree of short-range order is determined by the interaction strength, the central parameter in the creation of longrange order is the adsorbate's scattering phase. It determines whether $E(r)$ is repulsive at the distance $\sqrt{3}$ appearing as second-neighbor distance in a hexagonal lattice. It is seen from Fig. 4(a) that ordered chains, but only few trigonal units, are formed for $\mathrm{Co} / \mathrm{Cu}(111)$, since for its phase of $\pi / 2$, the distance $\sqrt{3}$ falls straight onto repulsion. Ideal formation of a hexagonal superlattice should be observed for $\delta=\pi / 5$, where the distance $\sqrt{3}$ is neutral. $\mathrm{Co} / \mathrm{Ag}(111)$ [Fig. 4(b)] comes closer to this with $\delta=\pi / 3$, however, its interaction 
strength is very weak and the energy minimum is very broad due to the small $k_{\mathrm{F}}$. As a consequence, a more isotropic adatom gas with short-range order is established. We note that $\delta$ is linked to the charge state of the adsorbates by Friedel's sum rule. ${ }^{22}$ Therefore it can possibly be tuned by coadsorption of electronegative or electropositive species, which should permit one to lock a preordered 2D gas into a lattice by increasing the $2 \mathrm{D}$ pressure.

In summary we have shown the existence of long-range adsorbate-adsorbate interactions for three metal/metal systems. Their mediation by Friedel oscillations of surface-state electrons expresses itself in the link of the oscillation period $\lambda$ of the interaction energy with the surface-state Fermi vector $\lambda=\pi / k_{\mathrm{F}}$. This relationship was demonstrated for $\mathrm{Cu}(111)$ and $\operatorname{Ag}(111)$, which have very different $k_{\mathrm{F}}$. Comparison between $\mathrm{Co}$ and $\mathrm{Cu}$ adatoms on $\mathrm{Cu}(111)$ shows, within the error margin, identical $E(r)$ curves, suggesting only little effect of the adsorbate's chemical identity on $E(r)$.
$\mathrm{On} \mathrm{Cu}(111)$ the predicted $1 / r^{2}$ decay is found from distances of $1 \mathrm{~nm}$ on. The adsorbate's scattering phase can be identified by application of a recent model showing excellent agreement with our data. For the systems investigated here, there is a significant short-range repulsion superimposed on the oscillatory long-range interactions. This short-range repulsion acts as an attachment barrier and delays island formation by which it changes nucleation kinetics to yield significantly enhanced island densities for a given ratio of the diffusion constant to deposition flux, $D / F$. The observed adsorbate-adsorbate interactions can potentially be employed for the creation of ordered atomic and molecular lattices. In the symmetry of such lattices the adsorbate's scattering phase and presumably also trio interactions play a central role.

We gratefully acknowledge discussions with $\mathrm{T}$. Cren and T. L. Einstein.
${ }^{1}$ T.L. Einstein, CRC Crit. Rev. Solid State Mater. Sci. 7, 261 (1978).

${ }^{2}$ G. Ehrlich and F. Watanabe, Langmuir 7, 2555 (1991).

${ }^{3}$ K.H. Lau and W. Kohn, Surf. Sci. 65, 607 (1977).

${ }^{4}$ J. Friedel, Nuovo Cimento, Suppl. 7, 287 (1958).

${ }^{5}$ T.F. Page and B. Ralph, Nat. Phys. Sci. 234, 163 (1971).

${ }^{6}$ H. Brune, J. Wintterlin, G. Ertl, and R.J. Behm, Europhys. Lett. 13, 123 (1990).

${ }^{7}$ K.H. Lau and W. Kohn, Surf. Sci. 75, 69 (1978). Note that the asymptotic $\cos \left(2 k_{\mathrm{F}} r\right) / r^{5}$ decay of this reference only holds for isotropic Fermi surfaces. In the general case, one must figure out which $\mathbf{k}$ vector along the Fermi surface to use. It is the one having its velocity in the direction of the interaction; see T.L. Einstein, ibid. 75, 161L (1978); T. L. Einstein, in Handbook of Surface Science, edited by W. N. Unertl (Elsevier Science, New York, 1996), Vol. 1, p. 578.

${ }^{8}$ J. Trost, T. Zambelli, J. Wintterlin, and G. Ertl, Phys. Rev. B 54, 17850 (1996); J.V. Barth, T. Zambelli, J. Wintterlin, R. Schuster, and G. Ertl, ibid. 55, 12902 (1997); S. Renisch, R. Schuster, J. Wintterlin, and G. Ertl, Phys. Rev. Lett. 82, 3839 (1999); L. Österlund, M.Ø. Pedersen, I. Stensgaard, E. Lægsgaard, and F. Besenbacher, ibid. 83, 4812 (1999).

${ }^{9}$ S.J. Koh and G. Ehrlich, Phys. Rev. Lett. 87, 106103 (2001); T. T. Tsong, Atom-Probe Field Ion Microscopy (Cambridge University Press, New York, 1990); G.L. Kellogg, Surf. Sci. Rep. 21, 1 (1994).

${ }^{10}$ O. Jeandupeux, L. Bürgi, A. Hirstein, H. Brune, and K. Kern,
Phys. Rev. B 59, 15926 (1999).

${ }^{11}$ N.W. Ashcroft and N.D. Mermin, Solid State Physics (HRW, Philadelphia, 1976).

${ }^{12}$ M.F. Crommie, C.P. Lutz, and D.M. Eigler, Nature (London) 363, 524 (1993).

${ }^{13}$ E. Wahlström, I. Ekvall, H. Olin, and L. Walldé, Appl. Phys. A: Mater. Sci. Process. A66, S1107 (1998).

${ }^{14}$ J. Repp, F. Moresco, G. Meyer, K.H. Rieder, P. Hyldgaard, and M. Persson, Phys. Rev. Lett. 85, 2981 (2000).

${ }^{15}$ A. Bogicevic, S. Ovesson, P. Hyldgaard, B.I. Lundqvist, H. Brune, and D.R. Jennison, Phys. Rev. Lett. 85, 1910 (2000).

${ }^{16}$ K.A. Fichthorn and M. Scheffler, Phys. Rev. Lett. 84, 5371 (2000).

${ }^{17}$ P. Hyldgaard and M. Persson, J. Phys.: Condens. Matter 12, L13 (2000).

${ }^{18}$ Full time sequences for all systems discussed in this paper can be found at http://ipewww.epfl.ch/gr_brune/Gallery/ gallery_b.htm and http://www.mpi-stuttgart.mpg.de/KERN/ Res_act/supmat_1.html

${ }^{19}$ S. Ovesson, A. Bogicevic, G. Wahnström, and B.I. Lundqvist, Phys. Rev. B 64, 125423 (2001).

${ }^{20}$ J.V. Barth, H. Brune, B. Fischer, J. Weckesser, and K. Kern, Phys. Rev. Lett. 84, 1732 (2000).

${ }^{21}$ M.M. Kamna, S.J. Stranick, and P.S. Weiss, Science 274, 118 (1996).

${ }^{22}$ J. M. Ziman, Theory of Solids, 2nd ed. (Cambridge University Press, Cambridge, 1972). 